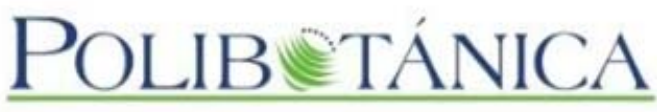

\title{
Polibotánica
}

ISSN electrónico: 2395-9525

polibotanica@gmail.com

Instituto Politécnico Nacional

México

http:www.polibotanica.mx

\section{EXPLORACIÓN ACTUAL SOBRE EL CONOCIMIENTO Y USO DE LA VAINILLA (Vanilla planifolia Andrews) EN LAS TIERRAS BAJAS MAYAS DEL NORTE, YUCATÁN, MÉXICO}

\section{CURRENT EXPLORATION ON THE KNOWLEDGE AND USE OF VANILLA (Vanilla planifolia Andrews) IN THE NORTHERN MAYA LOWLANDS, YUCATAN, MEXICO}

Rodríguez-López, T., y J. Martínez-Castillo.

EXPLORACION ACTUAL SOBRE EL CONOCIMIENTO Y USO DE LA VAINILLA (Vanilla planifolia Andrews) EN LAS TIERRAS BAJAS MAYAS DEL NORTE, YUCATÁN, MÉXICO.

CURRENT EXPLORATION ON THE KNOWLEDGE AND USE OF VANILLA (Vanilla planifolia Andrews) IN THE NORTHERN MAYA LOWLANDS, YUCATAN, MEXICO.

POlibetánica Núm. 48: 169-184 México. Julio 2019

cC (i) Este es un artículo de acceso abierto bajo la licencia Creative Commons 4.0 Atribución-No Comercial (CC BY-NC 4.0 Internacional). 


\title{
EXPLORACIÓN ACTUAL SOBRE EL CONOCIMIENTO Y USO DE LA VAINILLA (Vanilla planifolia Andrews) EN LAS TIERRAS BAJAS MAYAS DEL NORTE, YUCATÁN, MÉXICO.
}

\author{
CURRENT EXPLORATION ON THE KNOWLEDGE AND USE OF VANILLA (Vanilla \\ planifolia Andrews) IN THE NORTHERN MAYA LOWLANDS, YUCATAN, MEXICO.
}

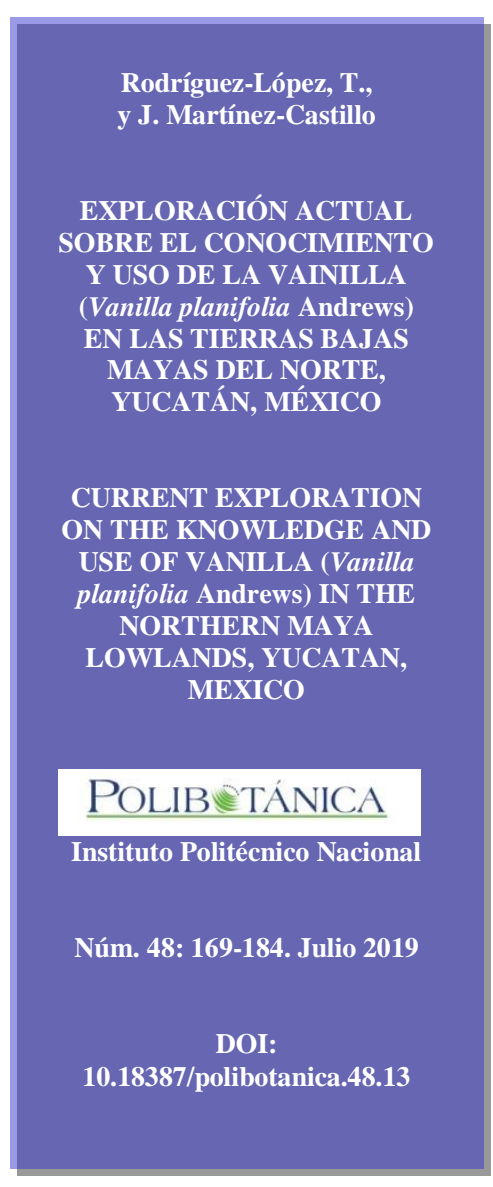

\author{
T. Rodríguez-López / teresita.rodriguez@uaem.mx \\ Departamento de Innovación Educativa. \\ Universidad Autónoma del Estado de Morelos,
} Av. Universidad 1001, Col. Chamilpa, CP 62209, Cuernavaca, Morelos, México.

J. Martínez-Castillo

Unidad de Recursos Naturales, Centro de Investigación Científica de Yucatán, A.C., Calle 43 núm. 130. Col. Chuburná de Hidalgo. 97205. Mérida, Yucatán.

RESUMEN: México es señalado como el área donde se inició el cultivo de la vainilla (Vanilla planifolia Andrews) a partir de poblaciones silvestres, siendo la región Totonaca del norte de Veracruz el área más probable. Trabajos antropológicos, señalan que los grupos mayas ubicados en la región cultural denominada Tierras Bajas Mayas conocían esta orquídea, nombrándola Sisbic. Sin embargo, no existen estudios sobre el conocimiento, uso y manejo de la vainilla por los pueblos mayas actuales. El objetivo de esta investigación fue recopilar el conocimiento y uso actual de la vainilla en 31 localidades de Yucatán y Quintana Roo pertenecientes a las Tierras Bajas Mayas reconocidas por la extracción del chicle. Se aplicó una encuesta a 130 parteras, el 48\% de ellas reconocen a la vainilla con 11 nombres vernáculos entre los que destacan el término Sisbic y cuatro usos principales: medicinal, ritual, perfume y saborizante. Dentro del uso medicinal, se reportó para atender 10 padecimientos, entre ellos, acelerar el parto, infecciones, depresión, dolor de cabeza, también se reportó el uso como planta ritual para elaborar una bebida usada por los $H$-menes y para adornos ceremoniales.

Palabras clave: vainilla, Sisbic, uso medicinal actual, Tierras Bajas Mayas del norte.

ABSTRACT: Mexico is designated as the area where the cultivation of vanilla (Vanilla planifolia Andrews) began from wild populations, with the Totonaca region of northern Veracruz being the most likely area. Anthropological works, indicate that the Mayan groups located in the cultural region denominated Mayan Lowlands knew this orchid, naming it Sisbic. However, there are no studies on the knowledge, use and management of vanilla by the current Mayan peoples. The objective of this research was to collect the knowledge and current use of vanilla in 31localities of Yucatan and Quintana Roo belonging to the Maya Lowlands recognized in the extraction of chewing gum. A survey was applied to 130 midwives, $48 \%$ of them recognize vanilla with eleven vernacular names, among which the term Sisbic stands out and four main uses: medicinal, ritual, perfume and flavoring. Within the medicinal use, it was reported to treat ten conditions, among them; accelerate childbirth, infections, depression, headache, also reported use as a ritual plant to make a drink used by the $\mathrm{H}$ men's and for ornaments.

Key words: Vanilla, Sisbic, current medicinal use, Northern Maya Lowlands. 


\section{INTRODUCCIÓN}

La vainilla (Vanilla planifolia Andrews) es una orquídea epifita, originaria de las selvas tropicales perennifolias de México y Centro América (Soto-Arenas, 2007); (Odoux, 2010); nativa de una franja de selvas húmedas en la vertiente del Atlántico (Golfo de México y el Caribe) desde Veracruz, Tabasco, Oaxaca, Chiapas y la Península de Yucatán en México (Villanueva Viramontes, 2017). Es la única orquídea aromática utilizada en la industria alimentaria y cosmética (Azofeifa-Bolaños, 2014), es considerada una de las plantas condimentarias de mayor precio del mundo, después del azafrán (Cardona-Medina, 2015). La vainilla se utilizó en la época prehispánica en Mesoamérica con varios propósitos: tributo, fragancia, rituales, saborizante del cacao y medicinal (Gamboa-Gaitan, 2014). Como sucede en muchos casos de plantas cultivadas, los mayores centros de cultivo y explotación están muy lejanos de los lugares de origen. Así, el principal productor de vainilla en el mundo es Madagascar, al este de África. En la península de Yucatán se ha reportado un nuevo género; Vanilla sp. nov. aff. V. Phaeantha, así como V. insignis Ames, las otras son: V. planifolia, V. odorata C. Presl. y $V$. inodora Schiede, crecen silvestres en reductos de selvas muy húmedas en centro y sur de la Península. Las comunes, en la Península son la Vanilla insignis y Vanilla sp. nov. aff. $V$. Phaeantha, la más rara (localmente) $V$. planifolia, aunque se han reportado reductos silvestres en la zona (Villanueva Viramontes, 2017). Estas plantas son de asociaciones vegetales estacionalmente secas y por ello bastante suculentas. Tiene los tallos finamente rugosos. Las hojas son oblongas. Los tallos son bastante rectos y sus entrenudos son muy largos y exceden el largo de las hojas. Las flores tienen un labelo de color verde más pálido que los pétalos y sépalos. La Vanilla planifolia, en cambio, es una planta de bosques húmedos y tiene hojas menos suculentas, aunque se reporta en lugares con bastante agua como cenotes (Villanueva Viramontes, 2017). Los tallos son lisos. Las hojas son elípticas, de forma que los márgenes foliares divergen desde la base hasta el centro, para luego hacer convergencia apicalmente. Los tallos se desarrollan en forma zig-zag y son más cortos que las hojas. Las flores, aun cuando similares a los de $V$. insignis y Vanilla sp. nov. aff. V. phaeantha son de color uniforme y el labelo tiene callos y márgenes diferentes (Carnevali, 2015; Villanueva, 2017).

Sobre su uso, documentos elaborados en el siglo XVIII, mencionan la primera evidencia histórica sobre el cultivo de los vainillales, en las regiones norte-centro de Colipa/Misantla y Papantla, en el estado de Veracruz, México (Bruman, 1948). En esa región, los grupos Totonacas nombraron a la vainilla como Axi-Xanath (flor recóndita) y desarrollaron la técnica de curado de esta vaina para perfumar la bebida de chocolate. Por ello, comúnmente se asigna el origen del cultivo de la vainilla a la cultura Totonaca (Barrera-Rodríguez, Herrera-Cabrera, Escobedo-Garrido, \& Bustamante, 2009); (Bory, 2008), no obstante, existen evidencias de recolección de vainilla y su cultivo proveniente de las Tierras Bajas Mayas (Roys, 1931), sus pobladores nombraban a la vainilla como sisbic-ak (bejuco oloroso), y era usada como medicina y perfume (Valencia, 2012); (Roys, 1931). En el Códice de la Cruz, del siglo XVI y XVII, se reportó para atender el parto, problemas del riñón y dolores de cabeza, dentro del uso ritual las semillas curadas de vainilla se mezclaban con resina de copal (Bursera sp. (L.) Sarg.), produciendo perfume utilizado en los templos sagrados, mientras que las vainas verdes se utilizaban para tratar las mordeduras de insectos venenosos y para curar heridas (Lawrence, 2001); (Bythrow, 2005). Francisco Hernández, en su relato de las propiedades medicinales de la vainilla, probablemente derivado de los médicos nativos mayas, mencionaba lo siguiente: "Una decocción de las vainas de vainilla en agua hace que la orina fluya admirablemente; mezclado con mecaxochitl (posiblemente flor de corazón) y vainas de vainilla causan aborto; también calientan y fortalecen el estómago; disminuyen la flatulencia; dan fuerza y vigor a la mente; curar problemas femeninos; y se dice que es bueno contra los venenos fríos y las picaduras de animales venenosos" (Olvera, 2019). El uso se reporta también asociado con el consumo de cacao (Theobroma cacao L.), especie con la que se elabora la bebida chocolate (Bruman, 1948) (Lawrence, 2001); (Barrera-Rodríguez, Herrera-Cabrera, Escobedo-Garrido, \& Bustamante, 2009) producto, proveniente del área Maya, siendo el chocolate de consumo exclusivo de la 
élite Maya y de los gobernantes Aztecas del Valle de México, quienes llamaban a la vainilla Tlilxochitl (flor negra), en lengua náhuatl (Bruman, 1948). Se ha señalado que la vainilla ha sido sometida a procesos de selección humana desde hace aproximadamente 3400 años por diversos grupos culturales Mesoamericanos, entre ellos los Mayas (Bruman, 1948); (Bory, 2008) (Lubinsky, 2008). Sin embargo, a pesar de su importancia económica como cultivo y su historia de manejo en el área Maya, no existen estudios etnobiológicos actuales y los inventarios florísticos de uso medicinal tampoco reportan el uso de esta especie en la zona maya. Por ello, el objetivo del presente trabajo fue recopilar el conocimiento, uso y manejo actual de la vainilla en las comunidades localizadas en la Tierras Bajas Mayas de México, así como discutir las estrategias de conservación y manejo de este recurso.

\section{MATERIAL Y MÉTODOS}

El objetivo de esta investigación fue recopilar el conocimiento y uso actual de la vainilla en las Tierras Bajas Mayas, utilizando un enfoque etnobiológico, así como el uso de técnicas etnográficas como la entrevista y la encuesta. Para identificar la especie se usaron fotografías y ejemplares vivos para su reconocimiento. Se realizaron las siguientes actividades; a) Recorrido en las Tierras Bajas Mayas, b) Identificación de las localidades en Yucatán cuyos nombres tuviera que ver con el nombre maya de la vainilla, c) Realizar entrevistas con las autoridades locales de Sisbichen, Sisbic, antropólogos, biólogos, historiadores y cronistas de la zona para identificar a las personas con información sobre el uso de la vainilla, de estas actividades, se concluyó que los médicos tradicionales o H-menes y parteras serían los informantes adecuadas conocer los usos actuales de la vainilla. Así la Jurisdicción Sanitaria Núm. 2 de Quintana Roo, quienes cuentan con un padrón de parteras, nos facilitaron la lista de tres municipios; Felipe Carrillo Puerto, José María Morelos y Othón Blanco (Salud, 2011-2016). La siguiente etapa, consistió en diseñar el recorrido para aplicar la encuesta a las parteras y utilizarlas para realizar una indagación cualitativa sobre el uso y conocimiento de la vainilla retomando los métodos de (Martínez-Salgado, 2012) y (Reyes-García, 2007). Se seleccionó como la población de estudio, al grupo de parteras, considerando que son personas que resguardan los conocimientos tradicionales sobre el uso de los recursos naturales y que aún conservan los rituales y costumbres trasmitidas de generación en generación.

Se realizaron cuatro rutas, recorridas en tres meses. Cada visita al domicilio de la partera resultaba adecuada sí ella se encontraba en su casa y aceptaba realizar la encuesta. La forma de acercarnos a ellas implicaba identificarnos con nuestros nombres, procedencia y el propósito de la visita, se solicitaba permiso para realizar la encuesta. La ausencia, muerte o declarar que era partera que usaba medicinas y no plantas, redujo la lista.

\section{Sitio de estudio}

Las Tierras Bajas Mayas, es una amplia región geográfica de influencia maya que abarcó desde la Península de Yucatán, hasta Guatemala. Según Chase, las Tierras Bajas Mayas pueden dividirse en dos regiones geográficas diferentes; Las Tierras Bajas del Norte, extendidas desde la Costa norte de Yucatán hasta Campeche (Loyola, 2008) caracterizadas por la presencia de vegetación más seca y menos precipitación pluvial y las Tierras Bajas del Sur, cuya característica principal es su abundante vegetación de selva alta perennifolia con cuantiosa precipitación y una orografía montañosa (Chase, 1985). La presente investigación se realizó en dos estados de la región que comprendieron las Tierra Bajas Mayas del norte, Quintana Roo y Yucatán. En Quintana Roo se abarcaron tres municipios; José María Morelos, Felipe Carrillo Puerto y Othón Blanco. Para Yucatán se trabajó en dos municipios Chichimila y Chemax, Las características de las 31 localidades encuestadas de zona de estudio son muy variadas en aspectos biológicos y sociales, el común denominador es la cultura maya, expresado en el manejo del idioma, vestimenta, tipo de vivienda, alimentación, rituales, en este estudio, las parteras que accedieron participar reconocieron la vainilla (Vanilla planifolia A), y se definían como parteras tradicionales ya que no usan medicina alópata. A continuación, se presenta el 
mapa 1 que ubica a las localidades encuestadas, así como la tabla 1 que menciona las características principales de ellas y la tabla 2 que resume el tipo de vegetación agrupada en cinco tipos distintos, según la base de datos que proporciona CONABIO.

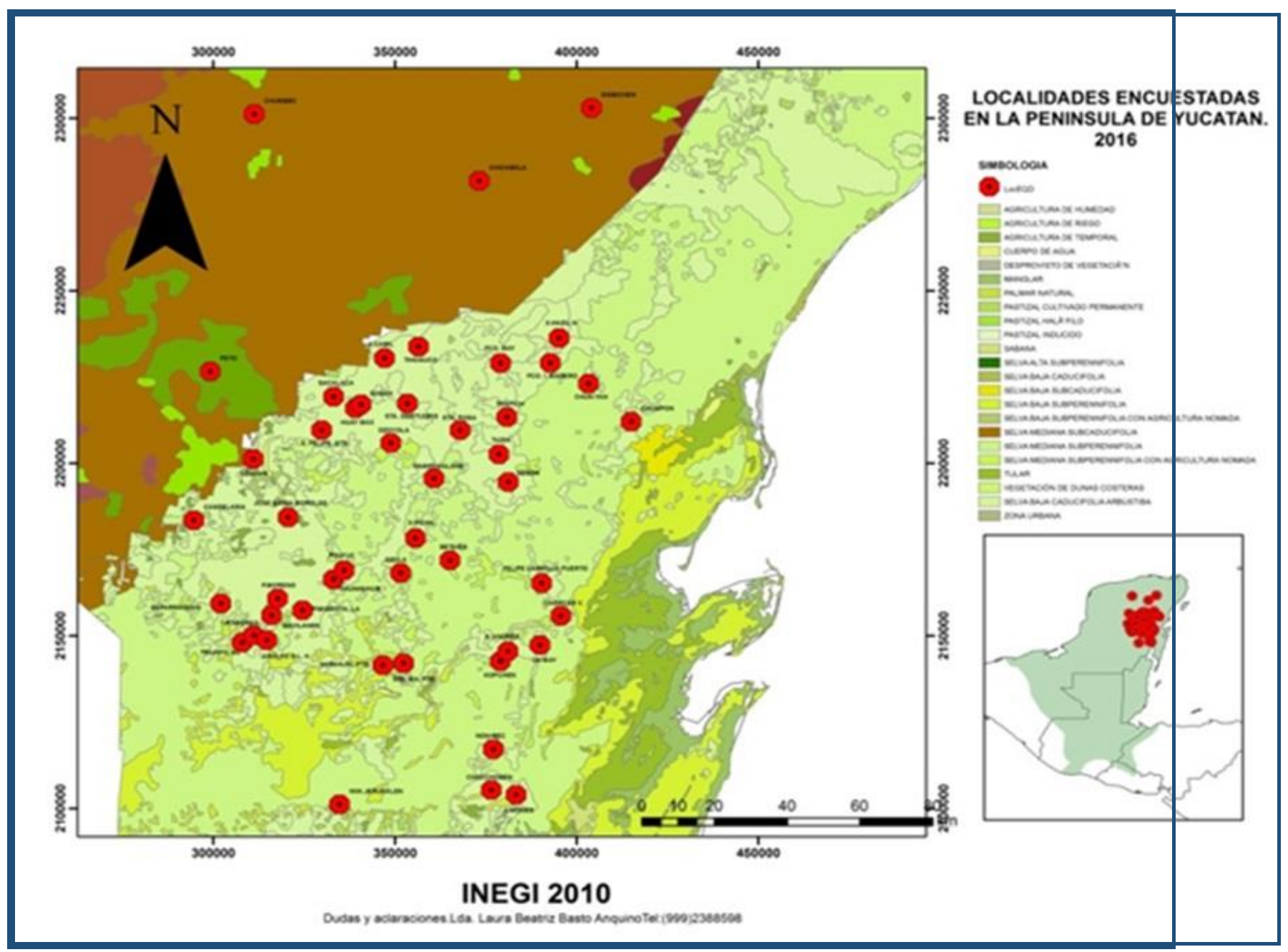

Fig. 1. Mapa de localidades en donde se encuestaron a parteras en la Península de Yucatán, 2016.

\section{Encuesta}

La encuesta fue conformada por 25 variables dividida en tres secciones. La primera sección se refirió a datos generales de la partera como su nombre, edad, lugar de nacimiento, actividad principal y quién le enseñó su actividad. La segunda sección se refirió al conocimiento sobre la vainilla, teniendo como apoyo a la planta en vivo y un juego de fotografías de Vanilla planifolia A. con tallo, flor, fruto y las vainas curadas. Finalmente, se preguntó si conocían la palabra "Sisbic" y sí sabían el significado. La aplicación de ésta se realizó con personas que hablan el maya, grabando de cada una de sus respuestas. Se tomaron fotografías de las parteras, sus familias y sus huertos. Las encuestas se vaciaron a una base de datos utilizando el programa estadístico de IBM SPSS Statistics versión 20. 
Tabla 1. Localidades encuestadas (31) y tipo de vegetación en donde sí conocen la planta.

\begin{tabular}{|c|c|c|c|c|c|c|}
\hline Estado & Municipio & Localidades & $\begin{array}{l}\text { Número de } \\
\text { habitantes }\end{array}$ & Ubicación GPS & $\begin{array}{c}\text { Altitud } \\
\text { (m s.n.m.) }\end{array}$ & $\begin{array}{c}\text { Tipo de } \\
\text { vegetación }\end{array}$ \\
\hline \multirow{13}{*}{ 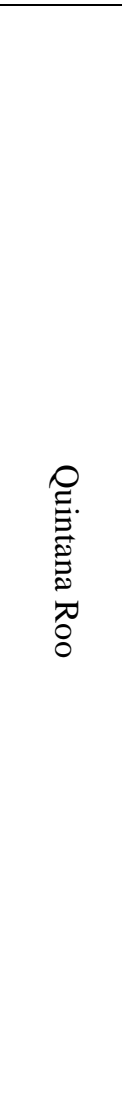 } & \multirow{13}{*}{ 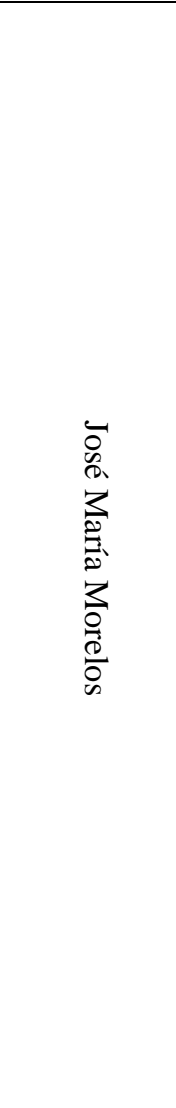 } & $\begin{array}{l}\text { Adolfo de la } \\
\text { Huerta }\end{array}$ & 97 & $\begin{array}{l}88^{\circ} 49^{\prime} 46.184^{\prime \prime} \mathrm{W} \\
19^{\circ} 25^{\prime} 5.794^{\prime \prime} \mathrm{N}\end{array}$ & 80 & 1 \\
\hline & & $\begin{array}{l}\text { José Ma. } \\
\text { Morelos }\end{array}$ & 9446 & $\begin{array}{l}88^{\circ} 42^{\prime} 45.301^{\prime \prime} \mathrm{W} \\
19^{\circ} 44^{\prime} 48.199^{\prime \prime} \mathrm{N} \\
\end{array}$ & 30 & 1 \\
\hline & & Pedro Moreno & 98 & $\begin{array}{l}88^{\circ} 44^{\prime} 14.803^{\prime \prime} \mathrm{W} \\
\text { 19³2'4.099”'N }\end{array}$ & 100 & 1 \\
\hline & & Gavilanes & 240 & $\begin{array}{l}88^{\circ} 45^{\prime} 12.954^{\prime \prime} \mathrm{W} \\
19^{\circ} 29^{\prime} 21.767^{\prime \prime} \mathrm{N}\end{array}$ & 80 & 1 \\
\hline & & Sacalaca & 1058 & $\begin{array}{l}88^{\circ} 35^{\prime} 45.064^{\prime \prime} \mathrm{W} \\
20^{\circ} 33^{\prime} 48.369^{\prime \prime} \mathrm{N}\end{array}$ & 30 & 1 \\
\hline & & $\begin{array}{l}\text { San Felipe } \\
\text { Oriente }\end{array}$ & 203 & $\begin{array}{l}88^{\circ} 4^{\prime} 15.15^{\prime \prime} \mathrm{W} \\
19^{\circ} 28^{\prime} 48.888^{\prime \prime} \mathrm{N}\end{array}$ & 30 & 1 \\
\hline & & Señor & 2362 & \begin{tabular}{|l|}
$88^{\circ} 8 ' 5.216^{\prime \prime} \mathrm{W}$ \\
$1^{\circ} 50 ' 38.029 ” \mathrm{~N}$
\end{tabular} & 25 & 1 \\
\hline & & Trapiche & 209 & \begin{tabular}{|l|}
$88^{\circ} 8 ' 23.59 ” \mathrm{~W}$ \\
$20^{\circ} 0$ \\
\end{tabular} & 30 & 1 \\
\hline & & Triunfo & 154 & $\begin{array}{l}88^{\circ} 45^{\prime} 53.832^{\prime \prime} \mathrm{W} \\
19^{\circ} 25^{\prime} 31.327^{\prime \prime} \mathrm{N}\end{array}$ & 70 & 1 \\
\hline & & Tuzik & 632 & \begin{tabular}{|l|}
$88^{\circ} 9^{\prime} 35.681^{\prime \prime W}$ \\
$1^{\circ} 54^{\prime} 58.086 ” \mathrm{~N}$
\end{tabular} & 25 & 1 \\
\hline & & X-Hazil Norte & 113 & $\begin{array}{l}88^{\circ} 0 ’ 13.835 ” \mathrm{~W} \\
20^{\circ} 13 ' 16.432 ” \mathrm{~N} \\
\end{array}$ & 30 & 1 \\
\hline & & Zafarrancho & 277 & \begin{tabular}{|l|}
$88^{\circ} 53^{\prime} 10.98^{\prime \prime} \mathrm{W}$ \\
$19^{\circ} 31^{\prime} 7.849 ” \mathrm{~N}$
\end{tabular} & 80 & 1 \\
\hline & & Pimientita & 231 & $\begin{array}{l}88^{\circ} 40^{\prime} 15.863^{\prime \prime} \mathrm{W} \\
1^{\circ} 30^{\prime} 12.766^{\prime \prime} \mathrm{N}\end{array}$ & 70 & 1 \\
\hline \multirow{11}{*}{ 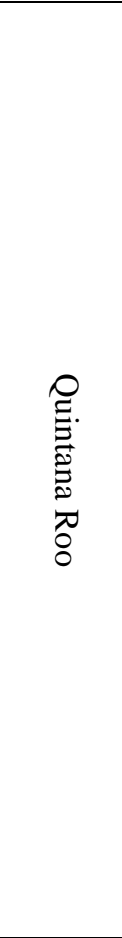 } & \multirow{11}{*}{ 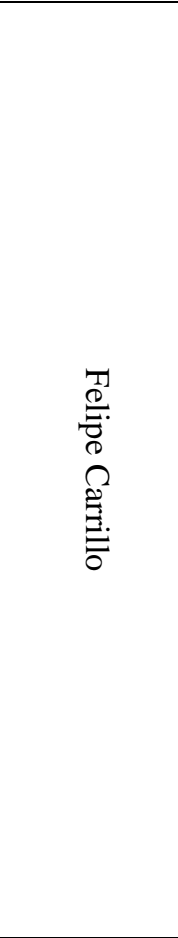 } & Betania & 497 & $\begin{array}{l}88^{\circ} 17^{\prime} 10.196^{\prime \prime} \mathrm{W} \\
19^{\circ} 38^{\prime} 14.436^{\prime \prime} \mathrm{N}\end{array}$ & 30 & 1 \\
\hline & & Candelaria & 810 & $\begin{array}{l}88^{\circ} 57^{\prime} 35.084^{\prime \prime} \mathrm{W} \\
1^{\circ} 44^{\prime} 12.418^{\prime \prime} \mathrm{N}\end{array}$ & 50 & 3 \\
\hline & & Chumpon & 481 & $\begin{array}{l}8^{\circ} 48^{\prime} 51.087^{\prime \prime} \mathrm{W} \\
20^{\circ} 00^{\prime} 15.02^{\prime \prime} \mathrm{N}\end{array}$ & 15 & 5 \\
\hline & & Chunya & 591 & $\begin{array}{l}8^{\circ} 555^{\prime} 33.591^{\prime \prime} \mathrm{W} \\
20^{\circ} 6{ }^{\prime} 7.447^{\prime \prime} \mathrm{N}\end{array}$ & 15 & 5 \\
\hline & & $\begin{array}{l}\text { Felipe Carrillo } \\
\text { Puerto }\end{array}$ & 18545 & $\begin{array}{l}88^{\circ} 2^{\prime} 42.646^{\prime \prime} \mathrm{W} \\
19^{\circ} 34^{\prime} 48.782 \text { 'N }\end{array}$ & 30 & 4 \\
\hline & & Huay-Max & 816 & $\begin{array}{l}88^{\circ} 31^{\prime} 24.846^{\prime \prime} \mathrm{W} \\
20^{\circ} 2^{\prime} 38.383^{\prime \prime} \mathrm{N}\end{array}$ & 30 & 5 \\
\hline & & Limones & 1882 & $\begin{array}{l}88^{\circ} 6^{\prime} 31.318^{\prime \prime} \mathrm{W} \\
19^{\circ} 1 ' 29.373 ” \mathrm{~N}\end{array}$ & 25 & 1 \\
\hline & & Naranjal & 17 & $\begin{array}{l}87^{\circ} 52^{\prime} 15.509^{\prime \prime} \mathrm{W} \\
1^{\circ} 50^{\prime} 35.673^{\prime \prime} \mathrm{N}\end{array}$ & 15 & 1 \\
\hline & & Polyuc & 1114 & $\begin{array}{l}88^{\circ} 33^{\prime} 53.285^{\prime \prime} \mathrm{W} \\
1^{\circ} 36^{\prime} 44.564^{\prime \prime} \mathrm{N}\end{array}$ & 40 & 1 \\
\hline & & San Andrés & 318 & $\begin{array}{l}88^{\circ} 8^{\prime} 0.908^{\prime \prime} \mathrm{W} \\
19^{\circ} 24^{\prime} 2.459^{\prime \prime} \mathrm{N}\end{array}$ & 25 & 1 \\
\hline & & \begin{tabular}{|l|} 
Sta. Rosa \\
(SEGUNDO)
\end{tabular} & 1010 & $\begin{array}{l}88^{\circ} 15^{\prime} 45.775^{\prime \prime} \mathrm{W} \\
1^{\circ} 58^{\prime} 43.299^{\prime \prime} \mathrm{N}\end{array}$ & 20 & 1 \\
\hline
\end{tabular}




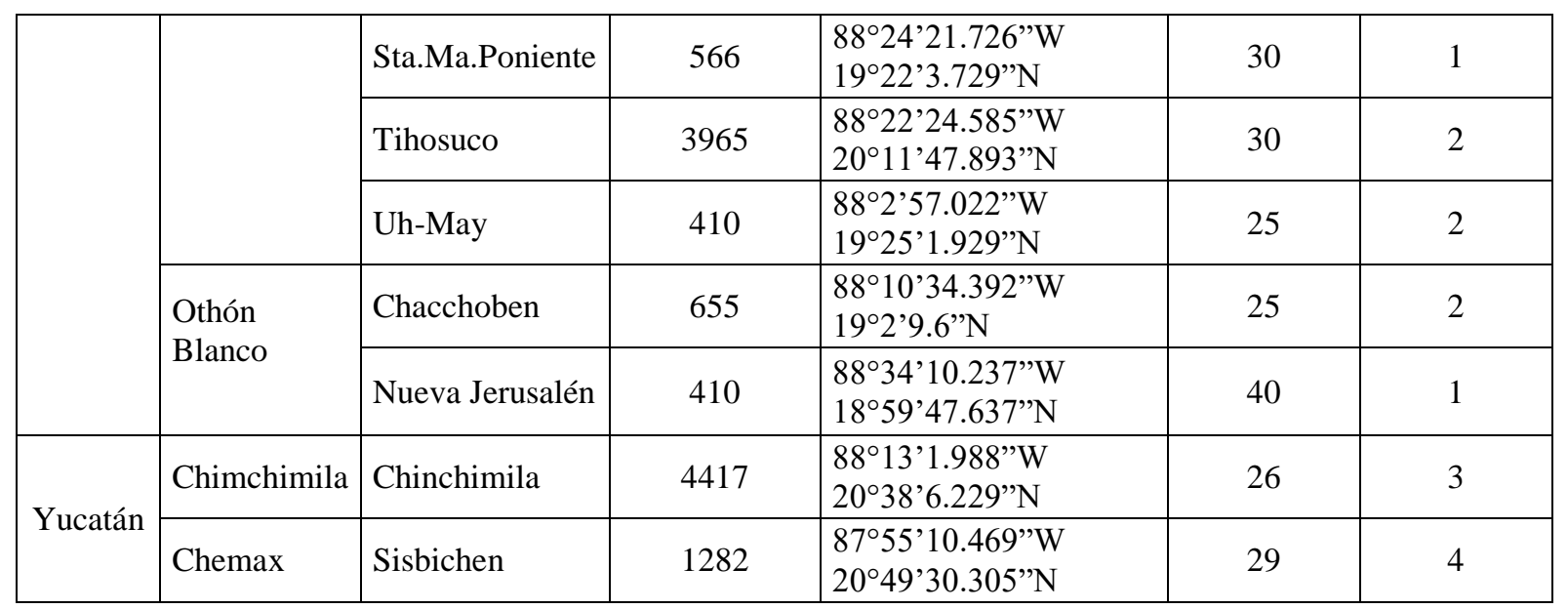

Fuente: Elaboración propia, 2019.

Como se puede apreciar en el mapa, la zona de estudio abarca la parte centro de Yucatán y Sur de Quintana Roo, a continuación, se presentan las características de cada localidad y su tipo de clima y vegetación.

Tabla 2. Tipo de vegetación, clima y precipitación pluvial para localidades encuestadas que conocen la vainilla (Vanilla planifolia A.) en las Tierras Bajas Mayas de Yucatán.

\begin{tabular}{|c|c|c|}
\hline $\begin{array}{l}\text { Identificador en tabla } \\
\text { de características de } \\
\text { localidades }\end{array}$ & Características climáticas & $\begin{array}{l}\text { Localidades con } \\
\text { este tipo de clima }\end{array}$ \\
\hline 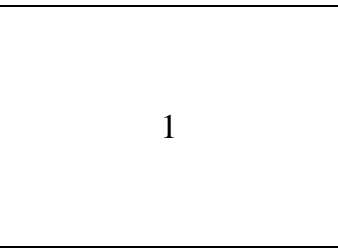 & $\begin{array}{l}\text { Vegetación secundaria arbustiva de selva mediana } \\
\text { subperennifolia con agricultura nómada, con } \\
\text { precipitación anual de } 800 \text { a } 1200 \mathrm{~mm} \text { clima cálido } \\
\text { subhúmedo, temperatura media anual de } 22^{\circ} \mathrm{C} \text { y } \\
\text { temperatura del mes más frío mayor a } 18^{\circ} \mathrm{C} \\
\left(\text { aw1 }\left(\mathrm{x}^{\prime}\right)\right) \text {. }\end{array}$ & 21 \\
\hline 2 & $\begin{array}{l}\text { Vegetación secundaria árborea de selva mediana } \\
\text { subperennifolia con agricultura nómada. clima } \\
\text { cálido subhúmedo, temperatura media anual mayor } \\
\text { de } 22^{\circ} \mathrm{C} \text { y temperatura del mes más frío mayor a } \\
18^{\circ} \mathrm{C}\left(\text { aw } 1\left(\mathrm{x}^{\prime}\right)\right) \text { con precipitación de } 800 \text { a } 1200 \mathrm{~mm}\end{array}$ & 4 \\
\hline 3 & $\begin{array}{l}\text { Agricultura de temporal. Clima subhúmedo, } \\
\text { temperatura media anual mayor de } 22^{\circ} \mathrm{C} \text { y } \\
\text { temperatura del mes más frío de } 18^{\circ} \mathrm{C}\left(\operatorname{aw} 1\left(\mathrm{x}^{\prime}\right)\right) \text {. }\end{array}$ & 1 \\
\hline 4 & $\begin{array}{l}\text { Selva mediana subcaducifolia. Clima subhúmedo, } \\
\text { temperatura media anual mayor de } 22^{\circ} \mathrm{c} \text { y } \\
\text { temperatura del mes más frío de } 18^{\circ} \mathrm{c} \text {. precipitación } \\
\text { de } 800-1200 \mathrm{~mm} \text { tipo de clima }\left(\mathrm{aw} 1\left(\mathrm{x}^{\prime}\right)\right)\end{array}$ & 2 \\
\hline 5 & $\begin{array}{l}\text { Selva mediana subperennifolia. Clima cálido } \\
\text { subhúmedo, temperatura media anual mayor de } \\
22^{\circ} \mathrm{C} \text { y temperatura del mes más frío mayor de } \\
18^{\circ} \mathrm{C} \text {, precipitación media anual de } 1200-1500 \mathrm{~mm} \\
\text { tipo de clima }\left(\text { aw } 1\left(\mathrm{x}^{\prime}\right)\right)\end{array}$ & 3 \\
\hline
\end{tabular}

Fuente: Elaboración propia, T.R.L., 2019. 


\section{RESUlTADOS}

Entrevistarse con Antropólogos, cronistas y autoridades locales que conocen la cultura maya, resultó ser un acierto, así la primer pista para realizar el trabajo la sugirió el doctor Briseño, quien apuntó que "Desafortunadamente la transformación de las Tierras Bajas Mayas en los últimos veinte años, ha provocado una pérdida de información sobre la cultura entre las poblaciones jóvenes, sin embargo, los grupos de curanderos o H-menes, así como las parteras han mantenido una organización particular que les permite resguardar su cultura" continuó mencionando "Hace veinte años, trabajar con parteras y H-menes era complicado, las investigaciones realizadas en la zona por diversos especialistas requerían de un largo tiempo para establecer un grado de confianza con los habitantes mayas, pues se sabe que resguardan sus conocimientos muy celosamente. Adentrar en rituales, o socializar la información de sus rituales está prohibido, incluso tomar fotografías durante estos procesos puede ser muy sancionado. Pero sin lugar a duda, sus conocimientos son muy importante para reconstruir un pasado fragmentado por diversos movimientos sociales, como la Guerra de Castas que termino en 1948 (Briseño, 2017).

El total de parteras registradas en la Jurisdicción Sanitaria núm. 1 fue de 130, se visitó a todas, pero solo se aplicó la encuesta a 82 parteras tradicionales de 31 localidades de Quintana Roo y Yucatán. 48 parteras eran muy grandes y estaban enfermas o habían fallecido o no se encontraban en su casa al momento de aplicar la encuesta. Se encuestaron a 37 parteras del municipio de José María Morelos y 31 de Felipe Carrillo Puerto, dos de Othón P. Blanco y 12 de Sisbic y Sisbicchen, Yucatán.

Del total de las parteras encuestadas, el $89 \%$ fueron mujeres y $11 \%$ hombres. La edad mínima fue de 44 años y la máxima de 99 años. Las parteras de edad muy avanzada solo hablan en maya, con quienes se utilizó traductor, expresándose en su idioma y respondiendo mucho mejor al ejemplar fresco que a las fotos. $47.6 \%$ del total reconocen la orquídea como vainilla o sisbic y tienen un rango de edad de 68 a 74 años. Son mujeres abuelas, que viven con sus hijos y nietos, algunas tienen su propia casa y viven cuidando su huerto, las más grandes están postradas en hamaca o cama, sin embargo, accedieron a responder la encuesta.

Tabla 3. Respuestas a la pregunta ¿Conoce esta planta? Por parteras de las Tierras Bajas Mayas, 2016.

\begin{tabular}{rlr|r|r|r}
\hline & Frecuencia & Porcentaje & \multicolumn{2}{c}{$\begin{array}{c}\text { Porcentaje } \\
\text { válido }\end{array}$} & \multicolumn{1}{c}{$\begin{array}{c}\text { Porcentaje } \\
\text { acumulado }\end{array}$} \\
\hline \multirow{2}{*}{ Válido } & $\mathrm{Si}$ & 39 & 47.6 & 47.6 & 47.6 \\
\cline { 2 - 6 } & No & 43 & 52.4 & 52.4 & 100.0 \\
\cline { 2 - 6 } & Total & 82 & 100.0 & 100.0 & \\
\hline
\end{tabular}

Fuente: Elaboración propia de base de datos. TRL. 2016. 
Gráfica 1. Porcentaje de edad de las parteras que conocen la Vanilla planifolia A. en las Tierras Bajas Mayas, 2016.

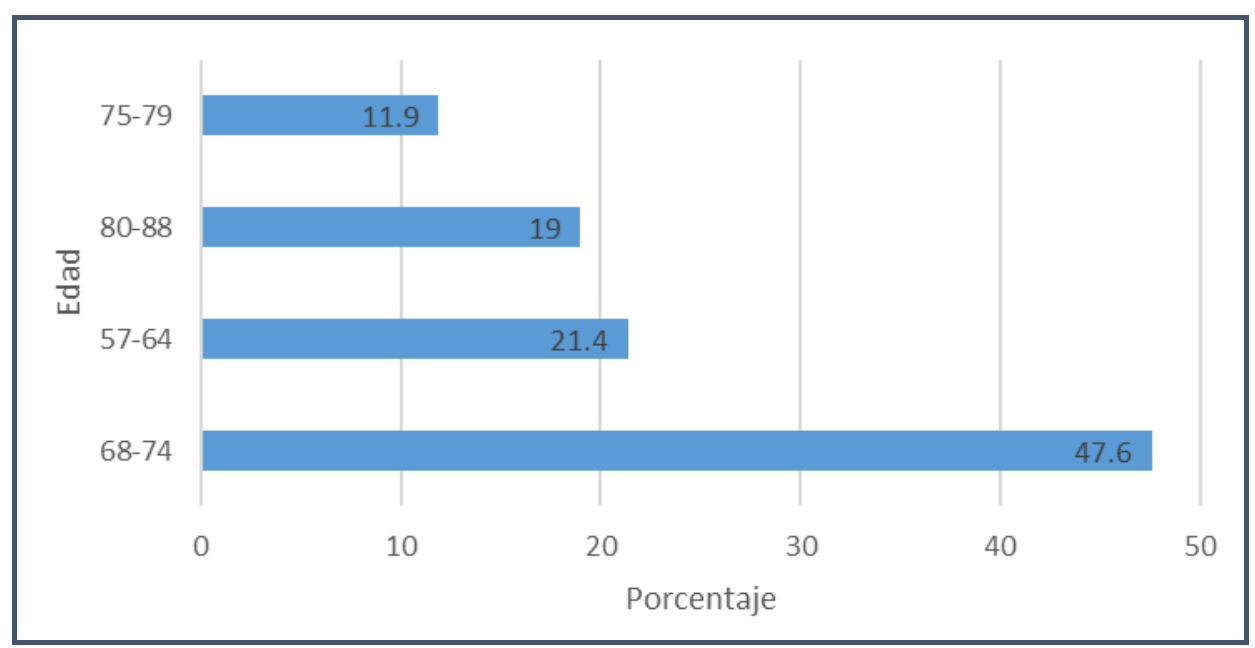

Fuente: Elaboración propia con la base de datos de las parteras de Tierras Bajas del Norte, 2016.

El problema que detectamos es para veinte de ellas, sus hijas o nietas no han querido continuar con su labor, ni quieren conocer sus tradiciones, las nuevas actividades de trabajo que se ofertan en la zona de Cancún no les permite continuar con el aprendizaje de las plantas.

Las principales actividades económica que ellas reportan son: $85 \%$ son parteras, $5 \%$ son curanderas, $4 \%$ respectivamente fueron hueseras y hierberas, $2 \%$ se dedica además a la agricultura y uno por ciento funge como auxiliar de salud, la mayoría además son amas de casa. Para el caso de los hombres, la actividad principal fue la de chiclero, es decir, la personas que extrae la sabía del árbol chico zapote (Manilkara zapota (L.) P. Royen) o chicle. Con menor porcentaje al uno por ciento para cada actividad, la reportaron ser carbonero, urdido de hamacas, auxiliar de salud y gestor. El gestor es aquel personaje que se dedican a apoyar a sus vecinos a realizar los trámites ante algunas dependencias de gobierno. Las personas que tienen sesenta a setenta años fueron quienes más recordaban la planta, uno de ellos, actualmente vive en el municipio de Felipe Carrillo Puerto, su esposa es partera. Relató que él fue chiclero en una etapa de su vida y reconoció la planta, la llamó orquídea o vainilla.

Sobre el lugar de nacimiento, 63\% nacieron en Quintana Roo y 35.4\% nacieron en Yucatán, solo $1 \%$ respectivamente para las que nacieron en Guatemala y Michoacán.

Sí nacieron en Yucatán, conocer el tiempo que tiene de vivir en Quintana Roo, puede indicar su relación con los recursos naturales y su conocimiento sobre los mismos, así 16 parteras tienen más de 50 años viviendo en la zona, 10 tienen 30-40 años, cuatro tienen 11-20 años y solo uno por ciento llevaban viviendo ahí 6-10 años, es decir, llevan poco tiempo de residencia en la zona, apenas una o dos generaciones, es posible que mantengan las costumbres del lugar de donde nacieron, efectivamente ellas reportan el uso de la vainilla como perfume y para usos rituales, luego los medicinales y finalmente, el uso más conocido es el saborizante.

De las parteras encuestadas en Quintana Roo y que conocen la planta, 27 nacieron en Yucatán y 11 en Quintana Roo, tal vez el conocimiento del uso de la vainilla como perfume proviene de Yucatán. 
Gráfica 2. Lugar de residencia de las parteras y frecuencia de encuesta en Tierras Bajas Mayas, 2016.

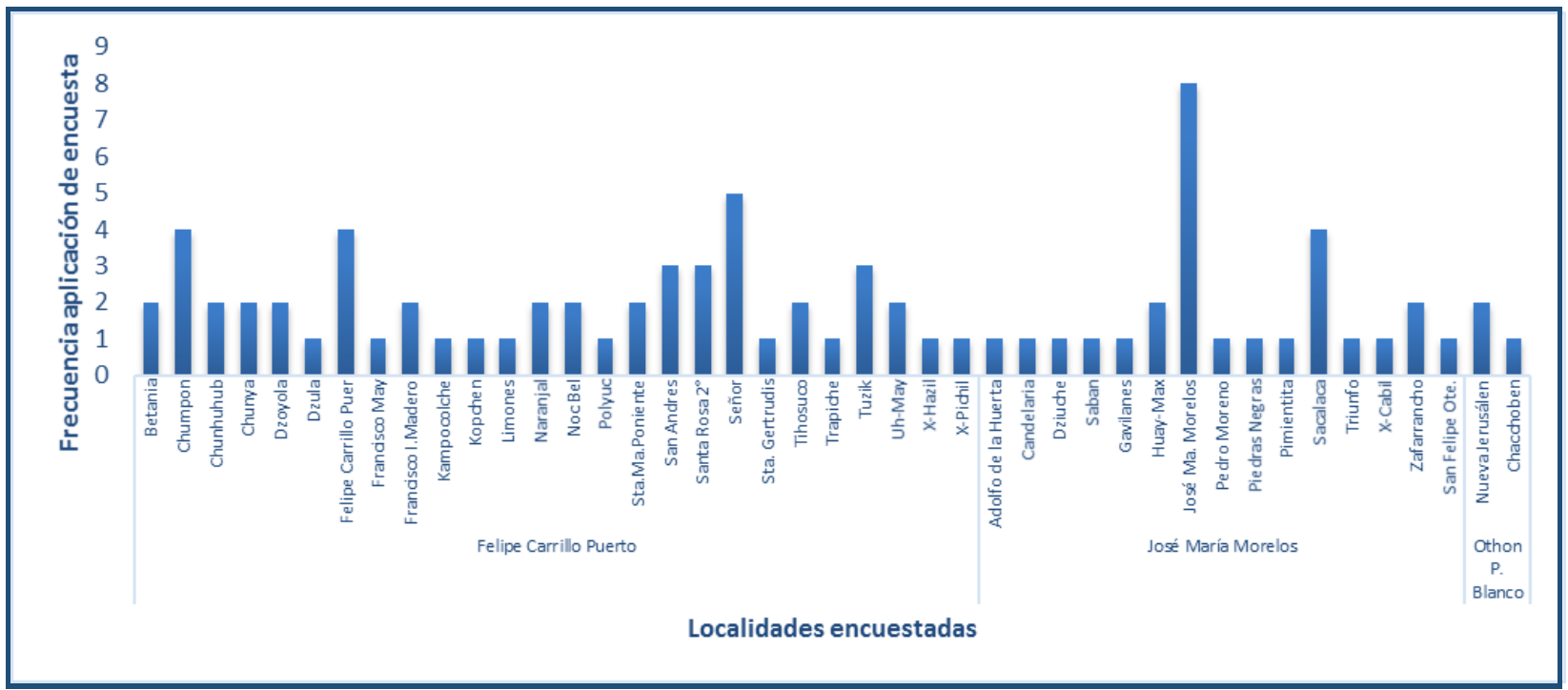

Fuente: Elaboración propia de base de datos de encuesta a parteras, 2016.

Gráfica 3. Frecuencia de lugar de nacimiento de parteras encuestadas, 2016.

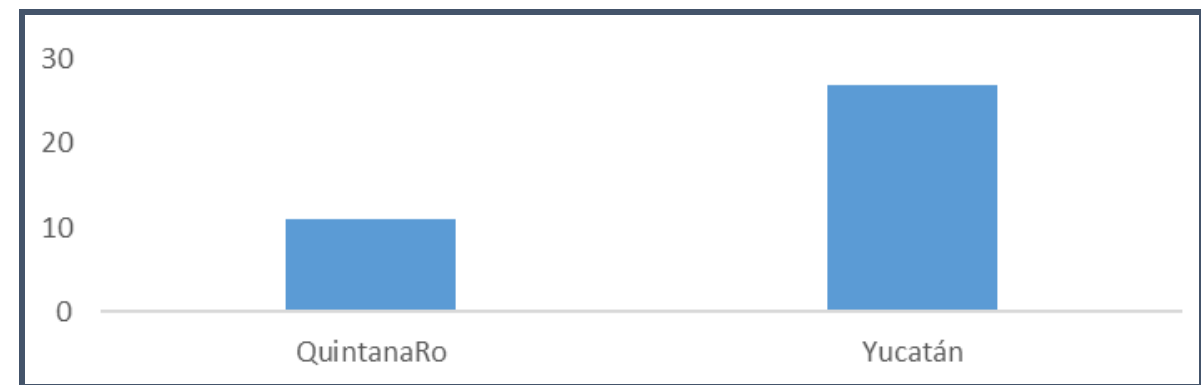

Fuente: Elaboración propia de base de datos de encuesta a parteras de las Tierras Bajas Mayas, 2016.

¿Qué significa sisbic, es la Vanilla planifolia?

Para un $4.9 \%$ de las parteras la palabra sisbic significa olor bonito y para el $2.4 \%$ es olor fresco, en maya es siss. Otra referencia que hacían era a sus abuelos o parientes de quienes escucharon el nombre, pero ellas comentaban que en la zona hay dos tipos de sisbic que huelen bonito. Tal vez son dos variedades de género Vanilla a la que las parteras reconocen como sisbic, ya en la introducción hacíamos referencia a que en la Península de Yucatán hay dos especies de vainilla olorosa, V. planifolia y V. sp. nov. aff. V. Phaeantha (Villanueva, 2017).

Se reconocieron doce nombres vernáculos distintos para la orquídea, la mayoría la reconoce como orquídea o vainilla, pero en algunos lugares le conocen con nombre mayas que hacen referencia a un bejuco que huele fresco. 
Tabla 4. Nombres vernáculos de la Vanilla planifolia A. reportados por las parteras ¿Cómo la nombra?

\begin{tabular}{|c|c|c|}
\hline Nombre maya & Significado & Frecuencia \\
\hline Sisbic ak & Bejuco oloroso & 7 \\
\hline Sisbic koc & Bejuco que emana fresco & 2 \\
\hline Xalalchec (totonaco) & Bejuco que huele fresco & 1 \\
\hline Lavo huech ó Labohuech, & Guía de chivo & 1 \\
\hline Siism & Fresco & 1 \\
\hline Caca ak & Bejuco con olor & 1 \\
\hline Huaco & Bejuco de agua & 1 \\
\hline Boc & Emana olor & 13 \\
\hline Ak-Chiu & Bejuco de olor & $\mathbf{3 1}$ \\
\hline tzuik & Que huele & 1 \\
\hline No recuerda el nombre, solo \\
vainilla u orquídea
\end{tabular}

Fuente: Elaboración propia de la Base de Datos, 2016.

A la pregunta de quién les había enseñado a usar la planta, el 90.2\% mencionó que un familiar, generalmente el abuelo(a) o padre quien también era curandero o partera. Solo un 5\% mencionó que, de algún compañero de trabajo, haciendo labores en el campo como chiclero, solo un $2.4 \%$ mencionó que "le mostraron la planta en sueño" el 2.4\% restante no contestó.

A la pregunta ¿Sabe en dónde crece la planta? el 93\% contestó que sí, al respecto se coloca la siguiente tabla 5 en dónde se preguntó sobre los sitios en los que crece la planta, para ubicar los sitios en donde la han visto, aunque hay que adherir las personas, $(2.4 \%)$ que mencionaron que no y un $4.9 \%$ no contestó. Intentando saber si recuerdan los lugares en donde han visto a la planta, se hizo la pregunta de si sabe en qué sitios crece la planta, las respuestas pueden apreciarse en la tabla siguiente:

Tabla 5. Sitios en donde las parteras ven comúnmente crecer a la orquídea.

\begin{tabular}{|l|c|}
\hline \multicolumn{1}{|c|}{ Sitios } & Porcentaje \\
\hline 1. Monte & 75.6 \\
\hline 2. Cenotes o Dzadz & 12.2 \\
\hline 3. Humedal & 7.3 \\
\hline 4. No contestó & 4.9 \\
\hline
\end{tabular}

Fuente: Elaboración propia, 2016. 
Una manera de conocer la percepción de las entrevistadas con respecto a la planta es preguntar si ellas cuando ven la planta la cuidarían y contrastarla con la siguiente pregunta si creen que la gente cuida este recurso. Los resultados nos arrojaron lo siguiente: 24 de 41 personas la quitarían, esto podría explicarse porque dentro de las actividades para preparar los terrenos para la siembra, que son de limpieza y preparación quitan todos los bejucos y plantas que se encuentran.

\section{Sobre los usos}

Sobre la pregunta ¿para que la usa? las parteras mencionaron lo siguiente; una mujer de 55 años, originaria de Valladolid, comenta que en el monte hay esta planta, que cuando acompañaba a su padre a sacar chicle había mucha, "la flor huele muy bonito" pero hay de hojas delgadas y otra de hojas más gruesas (posiblemente $V$. insignis y V. planifolia A.), las dos huelen bien, por eso su nombre "siss" que es olor agradable en maya, algo que huele bien y era usado como perfume. Comentó que desde que terminó lo del chicle hay menos sisbic en los montes. En otra localidad una partera de 89 años, originaria de Chimchimila, Yucatán, reconoció la orquídea, ella mencionó el nombre de sisbic, comentó que en su pueblo usaban la flor y los frutos para perfumar, cocían la flor con miel de abeja melipona, ahí bañaban su joyería, aretes, collares y con esa agua preparada se perfumaban, esta característica fue compartida por otra partera originaría de Peto, Yucatán. Una más, de 65 años, comentó que su abuelo paterno era H-men y su padre era curandero, ella tiene un gran conocimiento de las plantas usadas en rituales y sobre todo en las medicinales y esta orquídea se usa en aguas rituales actualmente entre los H-menes. Además de estos usos en rituales y como perfume, las parteras usan las vainas, tallos y flores en la atención de las siguientes enfermedades, que se han sistematizado en la tabla 6.

En la encuesta, las parteras mencionaron 4 usos, el $83 \%$ la reportó como medicinal, $10 \%$ como perfume, $5 \%$ como saborizante. Se reportó además que la vainilla ( $V$. planifolia A.) atiende diez padecimientos y es usada en la elaboración de aguas rituales que toman los H-menes y adornos en rituales para fiestas, se colocaban collares elaborados con vainas de la orquídea, la tabla de frecuencias nos arrojó la siguiente información.

El $34 \%$ de las parteras reconocen a la planta con calidad fría, es decir atiende problemas de naturaleza caliente, como la calentura por infección, el dolor de cabeza, el aire, sobresalen los siguientes usos y formas de uso como se aprecia en la tabla que a continuación se presenta.

Tabla 6. Usos medicinales de la Vanilla planifolia en las Tierras Bajas Mayas (2016).

\begin{tabular}{|c|c|c|c|}
\hline Padecimiento & Parte usada & $\begin{array}{c}\text { Forma de uso } \\
\end{array}$ & Frecuencia \\
\hline $\begin{array}{l}\text { Dolor de cabeza } \\
\text { Aire }\end{array}$ & hojas & $\begin{array}{l}\text { Se juntan dos hojas y con "vaporu” y sal, se pone en la cabeza y si se } \\
\text { derrite la sal, significa que está muy fuerte el dolor de cabeza }\end{array}$ & 12 \\
\hline Cicatrizante & flor & Se hace una pomada con la flor y se coloca en la herida & 4 \\
\hline Cáncer & $\begin{array}{l}\text { tallos y } \\
\text { rizomas }\end{array}$ & Hervidos los tallos y rizomas, se toma varios días en ayunas & 3 \\
\hline $\begin{array}{l}\text { Dolor de vientre o } \\
\text { cólicos }\end{array}$ & flores y vainas & $\begin{array}{l}\text { Se prepara una pomada con las hojas junto con la siempre viva y la } \\
\text { sábila y se unta en el vientre }\end{array}$ & 3 \\
\hline Acelerar parto & hojas & Se sancochan con aka' xiu y se colocan en el vientre & 2 \\
\hline Ombligo & flores & Cuando nace un niño o niña, se coloca su ombligo en agua de vainilla. & 1 \\
\hline Infecciones vaginales & flor & Hervida se toma en ayunas & 2 \\
\hline Piedra de riñones & raíz & Hervida se toma siete días en ayunas & 1 \\
\hline Vómito & flores & $\begin{array}{l}\text { Hervida cinco flores y tomado en ayunas sirve para manejar la nauseas } \\
\text { en embarazadas }\end{array}$ & 1 \\
\hline Depresión & flores & $\begin{array}{l}\text { Infusión de flores durante la luna nueva, en niños las flores se colocan } \\
\text { en pulseras, el olor los tranquiliza. }\end{array}$ & 1 \\
\hline Úlceras & vainas y flores & Infusión de flores & 1 \\
\hline
\end{tabular}

Fuente. Elaboración propia información de grabaciones y base de datos (2016). 


\section{Manejo}

Al hablar de manejo, se hace referencia a las prácticas culturales que los habitantes de una localidad aplican en el uso de la vainilla, desde su cuidado o protección en el monte, hasta la siembra en su huerto o solar, lo que es común entre las parteras. Encontramos tres tipos de manejo:

Manejo por programas de conservación. Muy cerca del municipio de Felipe Carrillo Puerto, se encuentra la comunidad de X-Hazil, Quintana Roo, ahí se observó una zona perturbada de selva mediana perennifolia, en donde se pudo localizar Vanilla insignis y V. planifolia, creciendo sobre árboles de zapote (Manilkara zapota (L) Royen), carrete (Vitex guameri Greenm), guayaba (Psidium guajaba L.), boob (Coccoloba coronata Jacq.), se sabe que antiguamente en esta zona se explotaba el chicle y cuando lo trabajaban quitaban las plantas que crecían sobre los árboles. Actualmente el sitio se encuentra dentro de un programa de manejo por la SEMARNAT-03ARN, lo que esté permitiendo la recuperación de la vegetación original y permitiendo que la vainilla se desarrolle libremente.

Manejo cultural. El denominador común en las parteras que reconocieron la planta como "Sisbic" es que sus familiares, padres y/o abuelos estaban relacionados con personas que explotaban el chicle y que fueron curanderos. Además, en la zona, hubo una época de extracción del chicle que duró hasta después de la segunda guerra mundial, y como sucede siempre con las plantaciones nativas que resultan ser económicamente viables a otros países, se llevan los recursos naturales y cambian las actividades productivas de la zona. Así que esta relación de la orquídea y sus habitantes se presentó entre los que extraían el chicle y los que la nombraban sisbic, y no tanto como se indicaba en la introducción, por el cultivo y extracción de cacao. Otro hecho que llama la atención es que las mujeres de la región maya, cuyo tipo de vegetación es más seca la conozcan con uso ritual y de perfume y las personas originarias de la zona de selva mediana no tenían este conocimiento. Una posible respuesta, es que Quintana Roo es un estado joven, y en 1974 hubo un programa de gobierno para que los habitantes de Yucatán, Peto, Chichimila trabajaran con el chicle de ahí el movimiento de las personas hacia las zonas de explotación del chicle y el conocimiento de la planta.

En el Poblado Sisbicchen es un lugar en donde se reconoce esta planta como ritual, Sisbichen (Lugar del sisbic, en lengua maya), fundado en 1841, durante el auge en la época del chicle (Yucatán, 1800-1900), es un poblado muy pequeño de 1747 habitantes (INEGI, 2010), cuenta con una escuela primaria y una telesecundaria. Una señora de 79 años, habitante del lugar, comentó que la abuela de su abuela, le decía:

"En este árbol, -una ceiba- había la planta sisbic y la flor lo hervía con miel y producía un olor agradable y lo untaban en los adornos de las mujeres, era perfume de fiestas y con el tiempo se lo acabaron y no lo sembraron" (Comunicación personal). Esta historia ha pasado ya tres generaciones en la familia de esta señora. La ceiba (Ceiba pentandra L.) es un árbol de importancia simbólica en la zona, representa un árbol sagrado de vida entre los mayas. En el poblado se encuentra este árbol, rodeado de piedras blancas y cuidado, pero ya no existe evidencia de los sisbiques. A las afueras del pueblo hay una Asociación Civil llamada Ykalakdzonot -el Jardín del Colibrí Dorado-, dirigido por un H-men, originario de Peto, en donde atienden aspectos relacionados con la salud y en su jardín de más de una hectárea y tres cenotes, cuenta con varias plantas de vainilla que encontró ahí mismo, no las sembró son plantas que ahí han crecido, silvestres, son cuatro de las cinco especies de vainilla, reportadas para la Península, este tipo de manejo se conoce como tolerancia por cultura "In situ", no hay tampoco una necesidad del rescate de la planta o de cultivarlo masivamente. Otra forma de manejo que se reportó fue la que hace la Asociación Civil en el municipio de Othón Blanco, Quintana Roo, aglutina a 10 comunidades, llamada "Unión regional de productores tradicionales Sisbic" con 200 socios activos y una superficie de 72 hectáreas, sin embargo, sembrar plantas de Veracruz, es continuar con el monocultivo y el empobrecimiento genético de la vainilla (fig. 2). 

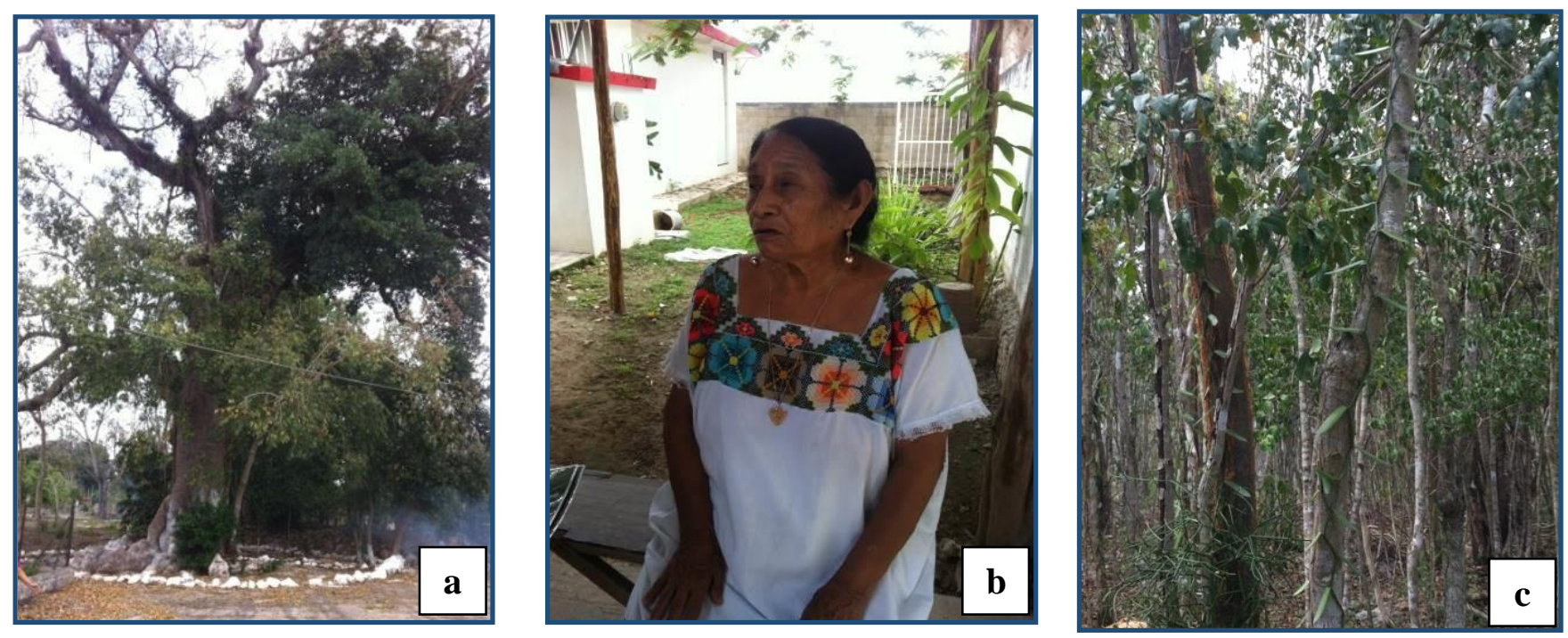

Fig. 2. Ceiba sagrada en Sisbichen, en dónde había sisbic usado como perfume (a). Partera de Sisbichen que reportó su uso como perfume y ritual (b). X-Hazil y Vanilla en campo (c). Fotos tomadas por T.R.L., 2016.

\section{Análisis de resultados}

La etnobiología cuantitativa, nos permite relacionar información individual y agruparla para encontrar los usos antiguos que los pobladores, por razones sociales, productivas, económicas y culturales, mantienen resguardadas de tal forma que las coincidencias de la información por un conjunto de personas cuya relación es solo la actividad social a la que se dedican, dan forma a un conocimiento cultural que debe reconocerse ampliamente.

Si bien es cierto que aparentemente hay una gran población de parteras, 130, en realidad las tradicionales son 85 y de estás solo el 3\% conocía la orquídea, se confirma que el conocimiento sobre este recurso está en peligro de desaparecer, aunque la buena noticia es que existen dos orquídeas aromáticas en la Península que no estaban reportadas y que su uso en la zona de las Tierras Bajas del norte, con vegetación más seca, es confirmado por estás parteras del Centro de Yucatán. El asociar a las parteras nacidas en Chichimilá y Sisbichen sobre su conocimiento del uso de la vainilla como perfume y ritual, puede explicar porque ahí existen poblaciones silvestres, ya que se encuentran protegidas in situ.

También de los resultados se desprende que cuando la actividad principal está cercana al entorno natural como chiclero, hierbero, el conocimiento de la planta es evidente. Pero cuando el agricultor no fue chiclero, solo trabajó el henequén o la milpa, su entorno no lo familiarizó con la orquídea, por lo que no reconoce la planta. Fenómenos como la migración, la trasmisión de saberes, la edad y el cambio en las actividades productivas están afectando el conocimiento sobre la orquídea.

\section{CONCLUSIONES}

El uso de la Vanilla planifolia en la zona, parece más asociado a la actividad de extracción de chicle, en donde el nombre de sisbic se popularizo entre los dueños de ranchos y chicleros, quienes nombraron así a sus ranchos o ejidos. La orquídea aromática tiene cuatro usos importantes: Medicinal, Perfume, Saborizante y Ritual. Predomina su uso medicinal para tratar 11 afecciones de salud. El uso como perfume es un conocimiento local que abarca la región maya de algunas localidades como Chichimilá, Peto y Valladolid en donde algunas personas 
conservan este conocimiento. La pérdida del conocimiento que las parteras resguardan será irreversible para rescatar información valiosa sobre el uso de los recursos de la zona, sin embargo, no lo están trasmitiendo a las generaciones jóvenes, ni estos parecen estar interesados en sus tradiciones. Por otro lado, los usos reportados desde el siglo XVI aún están vigentes, tan es así que las investigaciones actuales sobre las plantas para tratar Infecciones, Problemas reproductivos, dolor de cabeza y depresión. coincide con que, en los últimos años, se explora las propiedades de la vainilla como agente antioxidante, antimicrobiano, antiinflamatorio y anticancerígeno (Romero-Estrada et al., 2016) (Havkin-Frenkel \& Belanger, 2009) (Lopez, 2010). Sin embargo, los cultivos actuales presentan graves problemas de erosión génica, debido a que sus plantaciones son por clonaciones, ahora esto podría cambiar, ya que en la zona reportes de poblaciones silvestres, que podrían cambiar la situación, pero además en las Tierras Bajas Mayas, Vanilla planifolia no se ha cultivado y no se conoce la práctica del curado de la fruta. Esto podría explicar que las propuestas de cultivo intensivo no estén funcionando en la zona, por ejemplo, en las propuestas de plantaciones en Quintana Roo. Se han reportado experiencias organizativas con ejidatarios para hacer una Asociación de Productores de Vianilla Maya, pero esta menciona que los campesinos no tienen experiencia para realizar el curado del fruto. Se propone realizar actividades en dónde la población joven recupere su uso, así como fomentar y apoyar las actividades locales de conservación in situ, como Chumebc y Sisbichen y que los beneficiados sean las poblaciones del lugar y el país en general.

\section{AGRADECIMIENTOS}

Agradecemos a las parteras que nos proporcionaron su información. Mención especial para el CONACYT por su apoyo con la beca de posdoctorado, fundamentalmente al doctor Enrique Cabrero Mendoza por la oportunidad, así como a todos los investigadores del CICY, particularmente al doctor Jaime Martínez Castillo, quien siempre tuvo acertadas sugerencias y paciencia en la elaboración del trabajo. En especial también quiero agradecer el importante apoyo, siempre afable y respetuoso del Maestro Francisco Chan y Don Filos, además las estudiantes de biología Tatiana Chan y Karla Wendy quienes siempre nos acompañaron y ayudaron en la traducción durante las encuestas y entrevistas.

\section{LITERATURA CITADA}

Azofeifa-Bolaños, J. \&.-V.-G. (2014). Importancia y desafios de la Conservación de la Vanilla spp. (Orquidaceae) en Costa Rica. Agronomía Mesoamericana, 189-202.

Barrera-Rodríguez, A., Herrera-Cabrera, B. J.-V., Escobedo-Garrido, S., \& Bustamante, G. A. (2009). Caracterización de los Sistemas de producción de vainilla (Vanilla planifolia A) Bajo Naranjo y en Malla sombra en el Totonacapan. Tropical and Subtropical Agroecosystems, 10, 199-212. doi:https://dio.org/10.1080/09650799800200055

Bory, S. G. (2008). Genet Resour Crop Evol. doi:https://doi.org/10.1007/s10722-007-9260-3

Briseño, F. (08 de Febrero de 2017). Dr. en atropología. (T. R. López, Entrevistador)

Bruman, H. (1948). The Hispanic American Historical Review. HAR, 28(3), 360-376. doi:10.2307/2507753

Bythrow, J. (2005). Vanilla as a Medicinal Plant. Seminars in Integrative Medicine, 129-131. doi:https://doi.org/10.1016/j.sigm.2006.03.001

Cardona-Medina, M. R. (2015). Indagaciones acerca del azafrán de raíz (Escobedia grandiflora (L.F.) Kuntze, en Antoquia-Colombia: Una especie olvidada. Etnobiologia , 85-93.

Chase, A. F. (1985). Postclasic Temporal and Spatial Frames for the Lowland Maya; The Lowland Maya Postclassic, 9-22.

Gamboa-Gaitan, M. A. (2014). Vainillas colombianas y su microbiota; Diversidad, cultivo y microorgánismos endófiticos. Universitas Scientiarum, 287-300.

Henry, B. (1948). The Culture History of Mexican Vanilla.

INEGI. (2010). 
Recibido:

20/octubre/2018

Aceptado:

27/mayo/2019
Lawrence, M. (2001). Native Orchids of Oklahoma. Oklahoma Native Plant Record. doi:10.22488/okstate.17.100005

Loyola, G. (2008). La relación entre Yuxma, Yucatán y Cobá, Quintana Roo durante el clásico tardío (600-700/750 D.C.). Cholula: Universidad de las Americas Puebla.

Lubinsky, P. B.-P. (2008). Origins and dispersal of cultivated Vanilla (Vanilla planifolia Jacks, [Orchidaceae]. Economic Botany, 127-138.

Martínez-Salgado. (2012). El muestreo en investigación cualitativa. Principios básico y algunas controversias. Ciéncia \& Saúde Colectiva, 17(3), 613-619.

Odoux, E. (2010). Vainilla. Boca Ratón: CRC Press.

Olvera, H. F. (2019). Francisco Hernández; Historía de las Plantas de la Nueva España, 1942. México, México: UNAM. Obtenido de htpp://www.franciscohernandez.unam.mx

Reyes-García, V. M. (2007). Concepts and methods in studies measurin individual ethnobotanical knowledge. Journal of Ethnobiology, 182-203.

Roys, R. (1931). Ethnobotany of the Mayas. New Orleans: Universidad of Tulane.

Soto-Arenas. (2007). Recopilación y análisis de la información existente sobre las especies mexicanas del género Vanills. México.

Valencia, J. (2012). Plan Rector Sistema Producto Vainilla. Puebla: SAGARPA.

Villanueva Viramontes, S. (2017). Vanilla planifolia A, and its relatives in the mexican Yucatan Peninsula Systematic analyses with ISSR and its. Botanical Sciences, 95(2), 169-187.

Yucatán, A. H. (1800-1900). 